\section{Antitumor vaccines with punch}

In the quest for cancer immunotherapies, tumor-specific antigens are often regarded as tumors' Achilles' heels, in that they can potentially induce an immune cytotoxic response against tumor cells. Idiotypes on the Ig receptor of clonal B-cell malignancies are candidates for such tumor-specific antigens, but because they are "self"-derived, they fail to stimulate a hefty immune response. Kwak and colleagues describe how they produced an effective antitumor vaccine by fusing lymphoma Ig variable fragments to chemokines-small secreted proteins that induce potent inflammatory responses (pp. 226 and 253).

\section{Building better promoters}

The use of natural viral or cellular promoters to express a therapeutic gene has met with only limited success, as a result of low levels of transcription or inactivation of the promoter following transfer to the target tissue. Refusing to be limited to the collection of natural musclespecific promoters, Schwartz and colleagues decided to create synthetic promoters of their own (see pp. 224 and 241). By randomly mixing and matching four distinct promoter elements from known muscle-specific genes, they generated a library of synthetic shuffled promoters. Screening this library for tissue-specific expression, they succeeded in identifying a few promoters whose strength in differentiated muscle tissue greatly exceeded that of the natural promoters from which they were derived.

\section{Invading diagnostics}

The development of specific, sensitive, and simple diagnostic tests is central to molecular medicine. In this issue, Brow et al. describe a powerful new molecular tool for the analysis of the gene at the level of single nucleotides. (p. 292). They have exploited the exquisite sequence sensitivity of a Flap endonuclease from archaebacteria to allow it to report on single nucleotide changes in target genes.

Agricultural productivity has always been subject to natural environmental variables such

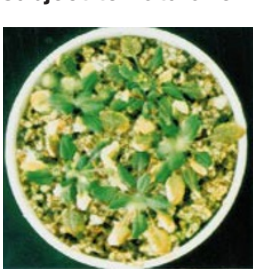
as drought, flood, or temperature extremes. In this issue, Shinozaki and colleagues describe a strategy to enhance a plant's resistance to a number of such environmental stresses, by conditionally expressing a transcription factor involved in upregulating a number of cellular stress proteins (pp. 229 and 287).

Research Briefs written by Natalie DeWitt and Robert Frederickson.

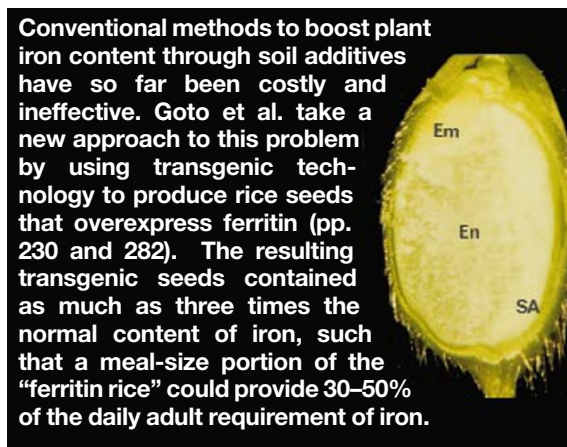

\section{A synthetic IL-10 mimic}

Small protein domains that bind to proteinprotein contact sites are important tools for drug discovery and biotechnology. But attempts to find bona fide "protein mimics" are often thwarted by the difficulty of making contacts between small linear molecules and flat protein surfaces. Reineke et al. tackle this problem using a synthetic, combinatorial approach to generate a discontinuous binding site on interleukin-10 that can bind tightly to its ligand, an IL-10 monoclonal antibody (p. 271).

Nucleoside antlviral drugs, such as AZT and acyclovir, must be phosphorylated by thymidine kinase before incorporation into DNA. Their effectiveness for antiviral therapies is based on the lower substrate affinity of the viral form of the enzyme, compared with the human form. In this issue, Christians et al. describe their use of a form of directed DNA evolution, called DNA family shuffling, to enhance the ability of the herpesvirus thymidine kinase to

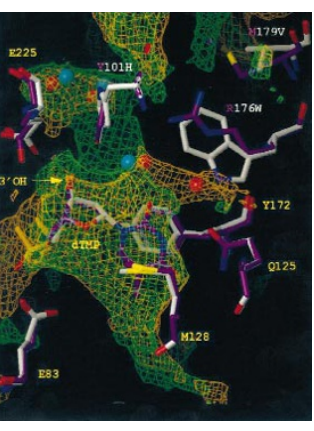

phosphorylate AZT (p. 259). The end product was a newly evolved "super" thymidine kinase, which had an increased ability to phosphorylate AZT, making Escherichia coli that express the enzyme susceptible to greatly reduced amounts of AZT.

\section{Stealthy RNases}

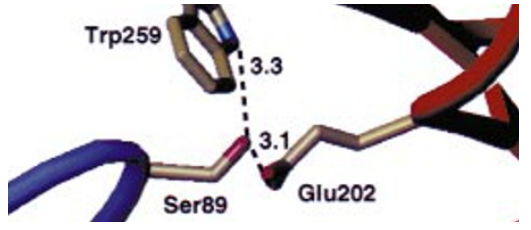

Targeting of toxic compounds to cells has been touted as a promising therapeutic approach to treat diseases such as cancer. The initial excitement gave way to a more sober view, however, as the targeted toxins failed to live up to their promise once tested in patients. Youle and colleagues now report an approach that uses an engineered form of the human body's own RNase protein (see pp. 227 and 265). These workers engineered the protein to target it to cancer cells and at the same time rendered it insensitive to the cytosolic ribonuclease inhibitor that normally limits its activity in cells. This stealthy feat of protein engineering resulted in a targeted therapeutic toxin 5,000 times more toxic to cancer cells than the normal RNase.

\section{Making antibodies more human}

Once heralded as magic bullets for cancer therapy, mouse monoclonal antibodies (MAbs) have failed to live up to their promise in clinical trials, partly because they elicit an immune response in humans. One way of making MAbs more tolerable to humans is to replace all but the variable regions essential for antibody specificity with human-derived regions. Usually this is complicated, involving transgenic mice and hybridoma technologies. But on page 276, Logtenberg and colleagues present a robust and time-saving new method for producing antitumor huMAbs from a phage library of displayed human single-chain antibody fragments.

\section{Reprogramming messages}

Gene therapy approaches that target either viral or defective cellular genes at the level of mRNA have focused on disruption of gene function, and include antisense technology to inhibit translation or use of an engineered ribozyme to cleave the message. In this issue, Puttaraju and colleagues have come up with a way of hijacking the cellular splicing machinery to actually repair genetic defects, and even reprogram gene expression, at the level of the message (pp. 223 and 246). Although not yet ready to be applied therapeutically, this work raises the tantalizing possibility that trans-splicing could one day be used as a platform to "rewrite" transcribed messages encoding disease-related genes. 